\title{
Implizites Wissen als Determinante des Kaufverhaltens produktbegleitender Dienstleistungen auf Industriegütermärkten - Eine explorative Analyse
}
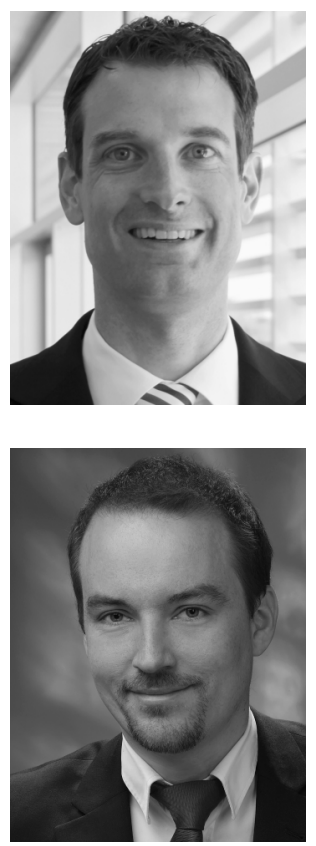

\section{Jens Hogreve und Richard Wonner}

Produktbegleitende Dienstleistungen, Erfolgsfaktoren, implizites Wissen, Unternehmenskultur, Dienstleistungsorientierung

Industrial Services, success factors, tacit knowledge, corporate culture, service orientation

Eine von Dienstleistungen getragene Wachstumsstrategie ist ein weit verbreitetes, jedoch selten erreichtes Ziel von Industriegüteranbietern. Bisherige Forschungsansätze untersuchen dieses Phänomen ausschliesslich aus Anbietersicht. Die vorliegende Studie befasst sich hingegen mit dem Kaufverhalten produktbegleitender Dienstleistungen aus Kundensicht. Die Ergebnisse zeigen, dass das Kaufverhalten stark von Situation und Unternehmenskultur des Kunden geprägt wird. Diesbezüglich kann gezeigt werden, dass implizites Wissen eine Schlüsselrolle einnimmt, da es den Wissensvorsprung des Anbieters schmälert und Kunden veranlasst, eigenes Personal zur Leistungserbringung aufzubauen.

Service-led growth is a common but rarely reached aim among industrial companies. However, current research analyses this important strategy mainly from a supplier perspective. Instead, this study sheds light on the buying behavior from a customer's point of view. The findings show that buying behavior is strongly determined by the customer's situation and corporate culture. In this context tacit knowledge turns out to be the main impediment for service sales as it diminishes the knowledge superiority of suppliers and leads customers to turn their corporate culture towards insourcing of services.

\section{Relevanz der Themenstellung}

Produktbegleitende Dienstleistungen sind im Industriegütersektor in den letzten Jahren verstärkt in den Mittelpunkt des Interesses von Wissenschaft und Praxis gerückt (Kunz) Hogreve 2011). Industriegüteranbieter nehmen zunehmend Dienstleistungen in ihr Portfolio auf und erweitern auf diesem Wege ihre Geschäftsmodelle (Oliva/Kallenberg 2003). Die Gründe für die Aufnahme eines Dienstleistungsgeschäftes sind vielfältig. Zum einen zielen Industriegüteranbieter darauf $\mathrm{ab}$, über das Angebot von produktbegleitenden Dienstleistungen eine signifikante Steigerung ihres Umsatzes und ihrer Erträge zu erhalten (Eggert et al. 2014). Auf diesem Wege können die Dienstleistungsumsätze rückläufige Ge- 
schäfte, die beispielsweise über grösseren Wettbewerbsdruck oder mangelnde Differenzierungsfähigkeit über das angebotene Produkt entstehen, kompensieren (Reinartz/Ulaga 2008). Zum anderen soll das Angebot von Dienstleistungen die Interaktion zwischen Kunde und Unternehmen verbessern, Kundenbindungsraten erhöhen und dadurch zusätzliche Informationen über die Kunden selbst generieren (Eggert et al. 2014). Diese Kundeninformationen wiederum können eine wertvolle Quelle für Angebotsinnovationen sein. Aus diesen Gründen werden industrielle Dienstleistungen in der Unternehmenspraxis und in der Wissenschaft als effektives Instrumentarium angesehen, das dabei hilft, Umsätze zu stabilisieren und Unternehmen weniger empfindlich gegenüber konjunkturellen Schwankungen werden zu lassen (Oliva/Kallenberg 2003; Fang et al. 2008).

Wagt man den Blick in die Unternehmenspraxis, so muss man jedoch feststellen, dass viele Unternehmen mit der Implementierung einer Dienstleistungsstrategie scheitern, wie der Fall von Hewlett-Packard zeigt. Im Rahmen eines Konzernumbaus hatte Hewlett-Packard intensiv in die Dienstleistungssparte investiert, um auf diese Weise Kundenbedürfnissen besser gerecht zu werden und insbesondere zusätzliche Erträge zu generieren. Da das Unternehmen die Kundenbedürfnisse zuvor jedoch nicht hinreichend analysiert und verstanden hatte, blieb die Investition in das Dienstleistungsgeschäft erfolglos. Die Verluste aus der Dienstleistungssparte beeinflussen heute die Umsatzrendite negativ (Bandler/ Burke 2012). Dass das Beispiel von Hewlett-Packard kein Einzelfall ist, zeigt eine Studie von Bain \& Co., welche nachweist, dass nur 21 Prozent der produzierenden Unternehmen Geschäftsmodellerweiterungen in den Dienstleistungssektor nachhaltig erfolgreich umsetzen können (Baveja et al. 2004). Gebauer et al. (2005) fassen die Beobachtung, dass das Angebot industrieller Dienstleistungen die an diese gerichteten hohen Erwartungen oftmals nicht erfüllen kann, unter dem „Service Paradox“ zusammen. Dabei beschreibt das „Service Paradox" eine Situation, in der eine expansive Dienstleistungsstrategie zwar zu steigenden Kosten, jedoch nicht zu den erwarteten Erträgen führt.

Die Forschung zum Angebot industrieller Dienstleistungen setzt bislang mehrheitlich an der Identifikation von Erfolgsfaktoren an (Eggert et al. 2011; Eggert et al. 2014). Gebauer et al. (2010) konnten in diesem Zusammenhang ermitteln, dass die Unternehmensorganisation einen bedeutenden Anteil am Dienstleistungserfolg in Industriegüterunternehmen hat. So ist es für das erfolgreiche Angebot von industriellen Dienstleistungen notwendig, dass die Organisation an die Besonderheiten von Dienstleistungen angepasst wird und somit einen hohen „Fit“ zur Dienstleistungsstrategie des produzierenden Unternehmens aufweist (Gebauer et al. 2010). Fang et al. (2008) untersuchen in einer vielbeachteten Studie den Zusammenhang zwischen dem Anteil des Dienstleistungsgeschäftes am Gesamtumsatz des Unternehmens und dessen Firmenwert auf der Basis von Längsschnittdaten. Sie kommen zu dem Ergebnis, dass die Implementierung einer Dienstleistungsstrategie unter Umständen auch negative Effekte auf die Unternehmensperformance haben kann. Dies gilt insbesondere für Unternehmen, die den Weg zum Dienstleistungsanbieter nur zögerlich beschreiten, und bei denen daher nur ein geringer Anteil des Umsatzes auf Dienstleistungen entfällt. Dies ändert sich jedoch signifikant, sobald ein Unternehmen einen Dienstleistungsanteil am Umsatz von 20 bis 30 Prozent überschreitet. Übersteigen die Dienstleistungsverkäufe diese Schwelle, hat das Dienstleistungsangebot einen positiven Effekt auf den Firmenwert.

Den Forschungsarbeiten über die Erfolgsfaktoren produktbegleitender Dienstleistungen ist gemein, dass sie die Wirkungen des Angebots industrieller Dienstleistungen ausschliess- 
lich aus der Anbieterperspektive untersuchen. Kundenseitige Faktoren, die den Dienstleistungserfolg beeinflussen könnten, sind bislang in der Literatur weitestgehend ausser Acht gelassen worden. Aus der Konsumentenforschung wissen wir jedoch, dass Kunden auf Dienstleistungsangebote sehr heterogen reagieren (vgl. z.B. Du et al. 2007). Ganesh et al. (2000) betonen in diesem Zusammenhang, dass Anbieter die Bedürfnisse ihrer Kunden genau kennen müssen. Dass dies auch im B2B-Kontext Geltung hat, unterstreicht auch das zitierte Beispiel von Hewlett-Packard. Homburg und Rudolph (2001) zeigen, dass Kunden auf Industriegütermärkten sehr spezifische Anforderungen haben, weshalb Anbieter ihre Produkte und Dienstleistungen stark den jeweiligen Kundenbedürfnissen anpassen müssen, um erfolgreich zu sein. Paulssen und Birk (2007) weisen nach, dass das Kaufverhalten industrieller Kunden stark von deren Kundencharakteristika beeinflusst wird. Diese Erkenntnisse zeigen, dass eine Betrachtung der Erfolgsfaktoren industrieller Dienstleistungen aus Kundenperspektive einen signifikanten Erkenntnisgewinn für die Dienstleistungsforschung darstellen könnte. So betrachten Antioco et al. (2008) die Erforschung der Erfolgsfaktoren des Angebotes industrieller Dienstleistungen aus Kundensicht als signifikante Lücke in der Literatur und als lohnenswertes zukünftiges Forschungsgebiet. An dieser Lücke setzt die vorliegende Studie an, indem sie qualitativ empirisch bedeutende Faktoren des Dienstleistungserfolges im B2B-Kontext identifiziert und analysiert. Mit den so gewonnenen Erkenntnissen werden zum einen die theoretischen Forschungen zum Angebot industrieller Dienstleistungen erweitert und zum anderen auch Implikationen für das nachhaltig erfolgreiche Management von B2B-Dienstleistungen gegeben.

\section{Zur empirischen Analyse des Angebots industrieller Dienstleistungen aus Kundensicht}

\subsection{Vorgehensweise und Sample}

Um die Frage nach den Erfolgsfaktoren des Angebotes industrieller Dienstleistungen aus Kundensicht beantworten zu können, wird in der vorliegenden Studie auf ein qualitatives Forschungsdesign zurückgegriffen, welches sich immer dann besonders eignet, wenn die zu beantwortenden Fragestellungen besonders explorativen Charakter aufweisen. Für die qualitative Studie wurden Leitfadeninterviews mit 28 Gesprächspartnern in 24 Unternehmen geführt. Die Gespräche fanden jeweils mit einem oder zwei Repräsentanten des Unternehmens statt und dauerten zwischen 40 und 90 Minuten. Insgesamt wurden 18 Stunden Tonmaterial auf 259 Seiten transkribiert. Die Auswertung des Datenmaterials erfolgte nach der Grounded Theory Methodik (Corbin/Strauss 2008).

Die Strichprobe umfasst auf Kundenseite 22 Gesprächspartner aus 18 produzierenden Unternehmen der Druck-, Elektronik-, Textil-, Kunststoff-, Pharma- und Automobilzulieferindustrie sowie der Lebensmittelverarbeitung. Der Umsatz der befragten Unternehmen beträgt zwischen 8 Mio. und 51 Mrd. EUR; die Mitarbeiterzahlen liegen zwischen 72 und 303.000 Beschäftigten. Die interviewten technischen Leiter, Produktionsleiter und Geschäftsführer waren jeweils die Entscheidungsträger der Unternehmen bezüglich des Dienstleistungseinkaufs und wiesen langjährige Erfahrung in der Bewertung und Koordination produktbegleitender Dienstleistungen auf.

Die kundenseitigen Interviews dienten der Erforschung der Determinanten des Kaufverhaltens bezüglich produktbegleitender Dienstleistungen. Um die Implikationen dieser Erkenntnisse für die Vertriebspraxis zu klären, wurden zusätzlich sieben Anbieterunternehmen aus den Bereichen Werkzeug-, Druck-, Verpackungs- und Lebensmittelmaschinenbau 
befragt. Die befragten Unternehmen beschäftigen zwischen 200 und 10.000 Mitarbeiter und erzielen einen Umsatz zwischen 10 Mio. und 5,5 Mrd. EUR. Fünf von den Interviewpartnern haben Positionen als Servicemanager, -direktor, oder -vorstand inne, zudem wurden ein Vertriebsleiter und ein Vertriebsmitarbeiter befragt. Die Interviews wurden semistrukturiert mit Hilfe eines Interviewleitfadens geführt. Zielsetzung der anbieterseitigen Interviews war dabei, die Sichtweise der anbietenden Unternehmen auf Kundendienstleistungen, die strategische Ausrichtung der Kundendienstpolitik sowie eine generelle Einschätzung des kundenseitigen Kaufverhaltens zu erforschen. Der Gesprächsleitfaden für die kundenseitigen Interviews war primär auf Fragen bezüglich der Unternehmenssituation und -organisation ausgerichtet, sowie deren Einfluss auf die Kaufentscheidungen der Kunden. Sowohl anbieter- als auch kundenseitige Interviews konzentrierten sich auf Dienstleistungen, die einerseits einen klaren Bezug zum verkauften Produkt aufweisen, jedoch andererseits separat von diesem verrechnet werden und somit ein eigenständiges Absatzobjekt darstellen. Demnach reichte das betrachtete Spektrum von klassischen Kundendienstleistungen wie Wartung und Reparaturen bis zu kundenorientierten Dienstleistungen wie Schulung, Consulting und Betreiberleistungen.

\subsection{Ergebnisse der qualitativen Studie}

\subsubsection{Der Einfluss impliziten Wissens auf die Kundenkompetenz}

Ein wesentlicher Erfolgsfaktor für das Angebot industrieller Dienstleistungen ist die Erkenntnis, dass für den effektiven und effizienten Einsatz von industriellen Produkten, wie beispielsweise Maschinen, explizites und implizites Wissen notwendig ist. Dieses Wissen wirkt sich wiederum direkt auf die Nutzung der produktbegleitenden Dienstleistungen aus. Zum einen ist explizites Wissen über die Funktionsweise einer Maschine erforderlich. Dieses Wissen über die Funktionsweise ist für die Erfüllung vieler Aufgaben jedoch nicht hinreichend. In der Regel ist zusätzlich implizites Wissen erforderlich, um den Einsatzgrad der installierten Maschine zu optimieren. Bei implizitem Wissen handelt es sich um nicht formalisierte Informationen, die sich aus dem betrieblichen Zusammenwirken von Mitarbeitern ergeben (Argyres 1996). Daher ist implizites Wissen über den betrieblichen Einsatzzweck der betreffenden Maschine oder Anlage sowie der Einbindung in Infrastruktur und Prozesse des Betreibers unabdingbar. In den Interviews wurde die Berücksichtigung beider Wissensarten bei der Erstellung industrieller Dienstleistungen als bedeutender Faktor auch für den Dienstleistungserfolg genannt.

Explizites Produktwissen ist primär bei produktbezogenen Arbeiten von Bedeutung. Hersteller profitieren dabei von beispielsweise im Produktentwicklungsprozess erworbenen Kenntnissen bezüglich der Funktionsweise und der Eigenheiten des Produktes (Raddats/Easingwood 2010).

Wie gesagt, [der Techniker des Herstellers] ist durchaus auch im Stande, in technischen

Problemfällen die Maschine schnell wieder zur Verfügung zu stellen. Da zeichnet sich auch der Vorteil eines Herstellers... ab. (Technischer Leiter einer Zeitungsdruckerei)

Je komplexer das gelieferte Produkt, desto mehr explizites Produktwissen ist für das Angebot begleitender, produktbezogener Dienstleistungen erforderlich. Insbesondere bei sehr komplexen Maschinen sehen sich die Kunden dadurch in einem grossen Abhängigkeitsverhältnis zum Hersteller, wenn es um die Nutzung begleitender Dienstleistungen geht: 
Bei manchen Maschinen ist es so, dass wir gar keine Wabl haben, weil die Maschine so komplex ist und dementsprechend so fein eingestellt, dass wir auf den Hersteller [zur Erfüllung von Aufgaben] angewiesen sind...

(Technischer Leiter eines Elektronikherstellers)

Bezogen auf den Dienstleistungsabsatz „konkurrieren“ die angebotenen Dienstleistungen jedoch mit Eigenleistungen des Kunden. Um anspruchsvolle Aufgaben an komplexen Maschinen und Anlagen ausführen zu können, ist ein hohes Mass an Expertise erforderlich, was eine intensive Schulung des Personals voraussetzt. Die Hersteller sehen in dem hierfür notwendigen Zeit- und Kostenaufwand einen Wettbewerbsvorteil für das eigene Leistungsangebot gegenüber einer Selbsterstellung durch den Kunden, da es für diesen meist nicht wirtschaftlich ist, das eigene Personal bis zu einem mit dem Herstellerpersonal vergleichbaren Kenntnisstand zu qualifizieren. Zudem haben Mitarbeiter des Herstellers Zugang zu technischem Wissen, welches exklusiv dem Hersteller zur Verfügung steht. Für den Dienstleistungsabsatz stellt dieses exklusive Wissen der eigenen Mitarbeiter einen Wettbewerbsvorteil dar.

Ich bin immer auf dem aktuellen Stand, was meine Produkte anbetrifft... wenn jemand Anderes unsere Baugruppen anfasst und ein Problem hat, der weiss nicht, dass wir da schon andere Lager drin haben und andere Buchsen usw.

(Senior Manager Service eines Werkzengmaschinenherstellers)

Es zeigt sich, dass produktbegleitende Dienstleistungen oft ein tiefgreifendes Verständnis des betreffenden Produktes voraussetzen, das zumeist nur der Hersteller hat. Die Erkenntnisse stützen damit die Aussage von Raddats und Easingwood (2010), wonach der Hersteller einen deutlichen Wettbewerbsvorteil gegenüber einer Selbsterstellung einer Dienstleistung durch den Kunden hat, da er viele Dienstleistungen effizienter oder exklusiv anbieten kann.

Anders stellt sich die Situation bezüglich des impliziten Wissens dar, das sich aus den betrieblichen Zusammenhängen ergibt. Um effizient mit den industriellen Produkten arbeiten zu können, ist tiefgreifendes explizites Produktwissen zwar notwendig, aber vielfach nicht ausreichend. Da die meisten Maschinen und Anlagen in unternehmensspezifische Prozesse und Abläufe eingebunden sind, ist die Kenntnis des Umfeldes und Einsatzzwecks elementar für eine effiziente Leistungserbringung.

Die Effizienz einer Fremdvergabe hängt immer davon ab, wie gut sich ein Lieferant bei uns auskennt. Ich kann nicht jedes Mal einen Neuen rein lassen, weil ein Drittel der Zeit lauf ich sonst neben dem her und sage "das darfst du nicht..., hier musst du aufpassen..."

\section{(Technischer Leiter eins Herstellers von Industriekeramik)}

Implizites Wissen betrifft sowohl die Einbindung der betreffenden Maschine oder Anlage in den Materialfluss als auch die Integration in die betriebliche Infrastruktur, wie beispielsweise die Anbindung an Versorgungs- und Entsorgungssysteme sowie an die IT-Infrastruktur. Hat der Hersteller beim expliziten Produktwissen einen Vorsprung gegenüber dem Kunden, so ist er bezüglich des impliziten Wissens oft im Nachteil, was den Dienstleistungsabsatz betreffend einen Wettbewerbsnachteil darstellt.

Das Mass, in dem das überlegene Produktwissen des Herstellers sich in einem Effizienzvorteil niederschlägt, hängt demnach stark von den Gegebenheiten beim Kunden ab. Er- 
fordert die Leistungserbringung ein hohes Mass an implizitem, kundenbezogenem Wissen, so relativiert dies den Kompetenzvorsprung des Herstellers (Argyres 1996). Aus Sicht des Herstellers verringert dies die Wettbewerbsfähigkeit des Dienstleistungsangebots gengenüber einer Selbsterstellung durch den Kunden.

\subsubsection{Der Einfluss impliziten Wissens auf Innen- und Dienstleistungsorientierung}

Die Interviewanalyse zeigt, dass in vielen der befragten Kundenunternehmen eine nach innen gewandte Leistungskultur (im Folgenden als Innenorientierung bezeichnet) vorherrscht. Innenorientierte Unternehmen vermeiden den Kauf von Dienstleistungen und präferieren stattdessen eine interne Leistungserbringung durch eigenes Personal. Die Gesprächspartner begründen diese Haltung damit, dass eigene Mitarbeiter durch die Ausführung von Tätigkeiten Maschinenwissen aufbauen. Damit steht im Problemfall das notwendige Wissen schnell und zuverlässig zur Verfügung, wohingegen beim Kauf von Dienstleistungen des Herstellers die Verfügbarkeit von Personalressourcen nicht sichergestellt werden kann.

Je spezifischer die Produktionsumgebung ist, desto stärker tendieren die betreffenden Kunden dazu, implizites Wissen und Routinen aufzubauen, die eine effiziente Arbeitsweise in der Leistungserbringung begünstigen (Kogut/Zander 1992; Grant 1996). Die Gesprächspartner betonen, dass sie im Vergleich zu Dienstleistungen des Herstellers deutliche Vorteile bei der Leistungserbringung durch das eigene Personal sehen. Diesbezüglich hilft das implizite Wissen um die Zusammenhänge im Unternehmen bei der effizienten Ausführung von Arbeiten.

...wenn irgendjemand von [A] kommt, der sieht nur seine Maschine... der ist da Spezialist, der findet den Fehler schneller... Wenn jetzt aber oben die Brandschutzklappe auch noch mit hängt - der sagt "das Ding oben blinkt" aber den interessiert es nicht... Wir versuchen unsere Leute ein bisschen zum Systemdenker zu erziehen... der weiss, wenn die Druckluft nicht geht, wo er hinlangen muss

(Technischer Leiter einer Zeitungsdruckerei)

Insbesondere bei ungeplanten Problemen befürchten die Interviewpartner eine unnötige Verlängerung von Produktionsausfällen, wenn zunächst Personal des Maschinenherstellers anreisen und in die Spezifika des Unternehmens eingewiesen werden muss.

Es gibt einen ganz einfachen Grund. OEE [(Overall Equipment Effectiveness)] muss hoch sein. Wann ist die OEE hoch? Wenn die Reaktionszeit kurz ist... Wann ist sie kurz - wenn die Spezialisten vor Ort sind... Das ist die Philosophie.

(Leiter Technische Dienste eines Automobilzulieferers)

Die Innenorientierung ist für den Absatz produktbegleitender Dienstleistungen von hoher Relevanz, da sie eine grundsätzliche Präferenz zum Aufbau eigener Ressourcen darstellt, die gewissermassen in "Konkurrenz" zum Dienstleistungsangebot des Herstellers stehen. Die Innenorientierung wird dabei wesentlich von der Verfügbarkeit von Wissen bestimmt. Kunden wollen durch den Aufbau eigener Ressourcen eine effiziente Erfüllung von Aufgaben durch Mitarbeiter sicherstellen, die über notwendiges implizites Produktionswissen verfügen. Da sich die Mitarbeiter am eigenen Standort befinden, ist zudem sichergestellt, dass im Problemfall schnell reagiert werden kann und somit Produktionsausfälle verhindert werden können. 
Die Analyse der Interviews zeigt, dass die Innenorientierung eng mit der Dienstleistungsorientierung der Kunden zusammenhängt. Innenorientierte Unternehmen betrachten die interne Leistungserbringung als für ihre Unternehmenssituation vorteilhaft. Mit einer innenorientierten Unternehmenskultur geht zumeist ein Aufbau eigenen Personals einher. Um diese Ressourcen effizient einsetzen zu können, müssen die Unternehmen eine nach innen gewandte Dienstleistungskultur entwickeln. Die Gespräche zeigen, dass damit insbesondere der Aufbau von Methoden zur objektiven Leistungsbewertung und ein entsprechendes Kostencontrolling einhergehen.

Wir sind im Moment ziemlich am Aufbauen von den ganzen Systemen... ich kann es im Moment noch nicht so spiegeln..., dass man sagt, die Maschine hat in diesem Jahr die und die Kosten gehabt, aber da sind wir, wie gesagt, gerade am Aufbauen.

(Technischer Leiter eines Elektronikproduzenten)

Dienstleistungsorientierung und die damit verbundene Kompetenz werden in den Kundenunternehmen nicht nur zur effizienten internen Leistungserbringung genutzt. Die befragten Unternehmen wollen damit auch zu einer objektiveren Einschätzung bei Make-or-Buy Entscheidungen bezüglich produktbegleitender Dienstleistungen gelangen. Eine starke Dienstleistungsorientierung der Kunden bedeutet für den Hersteller, dass der Kunde besser in der Lage ist, die erbrachten Dienstleistungen in Relation zu selbst erstellten Leistungen zu bewerten. Kunden mit hoher Dienstleistungsorientierung sind demnach für eine nutzenbasierte Produktpräsentation zugänglicher, sie sind jedoch auch eher in der Lage, entsprechende Nutzenversprechen der Hersteller zu überprüfen.

\subsubsection{Wirkung der Unternehmenskultur und Kompetenz der Kunden auf deren Kaufverhalten}

Die Interviews zeigen, dass dem Einkauf produktbegleitender Dienstleistungen eine Makeor-Buy Entscheidung vorausgeht, die primär von einem Vergleich des wahrgenommenen Kosten-Nutzen-Verhältnisses zwischen Selbsterstellung und Einkauf der Dienstleistung beeinflusst wird.

... da schauen wir sehr genau drauf. Auch das Insourcing, Outsourcing, das prüft man auch schon regelmässig, was kann man selbst machen,... unter einem Kostenaspekt ja, aber wirklich unter einem Risikoaspekt.

(Technischer Leiter eines Pharmaherstellers)

Die Kompetenz des Kunden beeinflusst dessen Kaufverhalten unabhängig von seiner Kosten-Nutzen-Wahrnehmung des Dienstleistungsangebots des Herstellers. Schätzen Kunden ihre eigene Kompetenz als vergleichbar oder gar überlegen gegenüber dem Hersteller ein, so führen sie Leistungen in der Regel auch selbst durch, ungeachtet der Attraktivität des Dienstleistungsangebots des Herstellers.

Einem erfahrener Automobilist, dem brauche ich nichts mehr über Instandhaltung sagen. Wenn er 100 Mann - was weiss ich - in seiner Instandhaltung hat, dann weiss er genau, wie er die aufgezogen hat

(Service Director eines Werkzeugmaschinenherstellers)

Fehlende eigene Kompetenz des Kunden mündet jedoch oft wie zuvor beschrieben in ein Abhängigkeitsverhältnis zum Hersteller. Ist dies der Fall, so muss der Kunde produktbe- 
gleitende Dienstleistungen beim Hersteller kaufen. Trifft dies zu, so ist die Wahrnehmung des Kosten-Nutzen-Verhältnisses seitens der Kunden für die Kaufentscheidung von untergeordneter Bedeutung, was zu einer „monopolartigen“ Absatzsituation für den Hersteller führen kann.

Weil die Anlagen so komplex sind, kann ich das nicht durch mein Instandhaltungspersonal abdecken - ist nicht möglich.

(Technischer Leiter eines Maschinenbauunternehmens)

Die Kompetenz des Kunden beeinflusst damit wesentlich dessen Kaufverhalten in Bezug auf produktbegleitende Dienstleistungen. Für Anbieter produktbegleitender Dienstleistungen sind daher besonders Situationen von Interesse, in denen Kunden weder über eine sehr hohe noch eine sehr niedrige Kompetenz verfügen, da in beiden Fällen das Kosten-NutzenVerbältnis für die Kaufentscheidung in den Hintergrund tritt. Verfügt der Kunde über sehr niedrige Kompetenz, so ist der Nutzen der Dienstleistungen des Herstellers offenkundig, sodass wenig Argumentation geleistet werden muss. Im Falle sehr hoher Kundenkompetenz lässt sich hingegen kaum ein Zusatznutzen der Dienstleistung gegenüber einer Selbsterstellung durch den Kunden identifizieren, sodass die Vorteilhaftigkeit des Angebotes durch den Hersteller nur über den Preis erzielt werden kann.

Im Gegensatz zur Kundenkompetenz, die direkt auf die Kaufbereitschaft der Kunden wirkt, beeinflusst die Unternehmenskultur der Kunden das Kaufverhalten indirekt, indem sie auf die Kosten-Nutzen-Wabrnehmung der Kunden wirkt.

[Die Preise sind] zu 90 Prozent zu hoch... [daher] wird es selber gemacht, was wir selber machen können

(Technischer Leiter eines Elektronikherstellers)

Ein wichtiger Aspekt der Unternehmenskultur betrifft die Innenorientierung der Kunden. Diese wirkt sich auf die Kaufbereitschaft der Kunden aus, da sie deren Kosten-NutzenWahrnehmung beeinflusst. Hält der Kunde für die Leistungserbringung eigenes Personal vor, so entstehen durch dessen Einsatz mittelfristig keine zusätzlichen Kosten. In der Folge schätzen Unternehmen mit starker Innenorientierung das Kosten-Nutzen-Verhältnis der vom Hersteller angebotenen Dienstleistungen als vergleichsweise unattraktiv ein. In Übereinstimmung mit Raddats und Eastingwood (2010) betrachten Unternehmen mit starker Innenorientierung die Erbringung vieler Leistungen als Teil ihrer Kernkompetenz und stellen daher eine schwer erreichbare Zielgruppe dar. Unternehmen mit weniger stark innenorientierter Unternehmenskultur greifen entweder generell auf Dienstleistungen des Herstellers zurück oder nutzen diese, um bei Ressourcenengpässen zu entlasten.

...also meistens ist es so, dass wir es intern lösen, weil wir selber einen Werkzengbau haben... Bei manchen grösseren Projekten ist es so, dass man das an den gleichen Hersteller oder einen Drittanbieter gibt. weil..., wenn wir jetzt noch einen grösseren Werkzeugbau machen, dann muss der auch ständig ausgelastet sein.

(Technischer Leiter eines Elektronikproduzenten)

Für die Kosten-Nutzen-Wahrnehmung des Kunden ist es von entscheidender Bedeutung, in wie weit der Kunde die Kosten für die Selbsterstellung einer Leistung korrekt bewerten kann. Die Analyse der kundenseitigen Interviews zeigt, dass die Kosten hierfür auch bei vergleichbarer betrieblicher Kostenstruktur beim Nachfragerunternehmen höchst unter- 
schiedlich eingeschätzt werden. Für den Absatz von Dienstleistungen stellt die hieraus resultierende mangelnde Objektivität der Kunden bei Entscheidungen bezüglich des Kaufs produktbegleitender Dienstleistungen ein Problem dar, da die Kunden zumeist die Kosten der Selbsterstellung unterschätzen.

Der Kunde wird an dieser Stelle nicht objektiv sein, weil er nicht objektiv Gleiches mit Gleichem vergleicht, denn seine Leute sind einfach da, und da machen viele Kunden den Fehler, wenn ich jetzt einen gewissen Part vergleichen will, dann rechne ich nicht alles rein.

(Personal Sales Manager After-Sales-Produkte eines Verpackungsmaschinenherstellers)

Die Analyse der Interviews zeigt zudem, dass die Dienstleistungsorientierung der Kundenunternehmen einen starken Einfluss auf deren Objektivität bei der Beurteilung des KostenNutzen-Verhältnisses von Dienstleistungen hat. Unter Dienstleistungsorientierung wird in diesem Zusammenhang die gezielte Ausrichtung der Unternehmenskultur auf eine effektive und effiziente Dienstleistungserbringung verstanden (Antioco et al. 2008). Dienstleistungsorientierte Unternehmen verfügen über Methoden zur Bewertung selbst erbrachter und fremdbezogener Dienstleistungen sowie zur Kostenkontrolle. In der Folge beurteilen Nachfrager mit ausgeprägter Dienstleistungsorientierung Leistungen ganzheitlicher und basieren die Entscheidung über den Kauf weniger auf dem Preis.

Servicequalität in der Vergangenheit wird durchaus auch in einem Punktesystem... zusammengefasst. Da ist der Preis sicherlich mit drin, das ist keine ganz unwesentliche Komponente, es sind aber alle anderen Komponenten auch mit drin. Wir bewerten Reaktionszeiten, ...das Vertrauen, ... die bisherigen Serviceeinsätze und natürlich das technische Konzept. Aber der Preis ist nicht das allein Entscheidende.

(Technischer Leiter eins Herstellers von Industriekeramik)

Die Interviewanalyse zeigt, dass das Kaufverhalten bezogen auf produktbegleitende Dienstleistungen stark von der Unternehmenskultur des Kunden abhängt. Insbesondere die Innen- und die Dienstleistungsorientierung sind dabei von zentraler Bedeutung für das wahrgenommene Kosten-Nutzen-Verhältnis der angebotenen produktbegleitenden Dienstleistungen und damit für das Kaufverhalten der Kunden. Interessant ist hierbei, dass die Innenorientierung zwar einerseits einen negativen direkten Effekt auf die Kosten-NutzenWahrnehmung hat, andererseits aber auch die Dienstleistungsorientierung der Kundenunternehmen fördert, was wiederum einen positiven Effekt auf die Kosten-Nutzen-Wahrnehmung hat (vgl. Abbildung 1).

Hieraus lässt sich folgern, dass eine starke Innenorientierung zwar zunächst negativ auf die Kosten-Nutzen-Wahrnehmung der Kunden wirkt. Andererseits unterstützt sie jedoch die Dienstleistungsorientierung, was zu einer objektiveren Beurteilung des Leistungsangebots führt. Ein entsprechender Zusammenhang lässt sich auch in den Interviews beobachten. So zeigen diese, dass Kunden mit starker Innenorientierung aktiv die Dienstleistungsorientierung im Unternehmen fördern, was zu einer zunehmenden Akzeptanz von produktbegleitenden Dienstleistungen des Herstellers führt. Die Analyse der Gespräche deutet darauf hin, dass dies auf eine zunehmende Professionalisierung seitens der Kunden zurückzuführen ist, die sich in einer vermehrten Dienstleistungsorientierung niederschlägt. Als Folge beurteilen die Nachfrager die angebotenen Dienstleistungen objektiver im Vergleich 
zur Eigenleistung, was zumeist zu einer vorteilhafteren Einschätzung des Kosten-NutzenVerhältnisses und somit zu einer grösseren Kaufneigung führt.

\section{Diskussion}

\subsection{Theoretischer Beitrag der Studie}

Die Erkenntnisse der Studie zeigen, dass der Erfolg produktbegleitender Dienstleistungen nicht nur, wie vorausgegangene Studien darstellen (vgl. z.B. Fang et al. 2008; Gebauer et al. 2010), von der anbieterseitigen Dienstleistungsstrategie bestimmt wird, sondern auch von spezifischen kundenseitigen Gegebenheiten abhängt. Aus den Ergebnissen lässt sich ein Modell kundenseitiger Determinanten des Kaufverhaltens produktbegleitender Dienstleistungen ableiten (vgl. Abbildung 1). Dabei werden drei wesentliche Beiträge zur Theorie der produktbegleitenden Dienstleistungen geleistet.

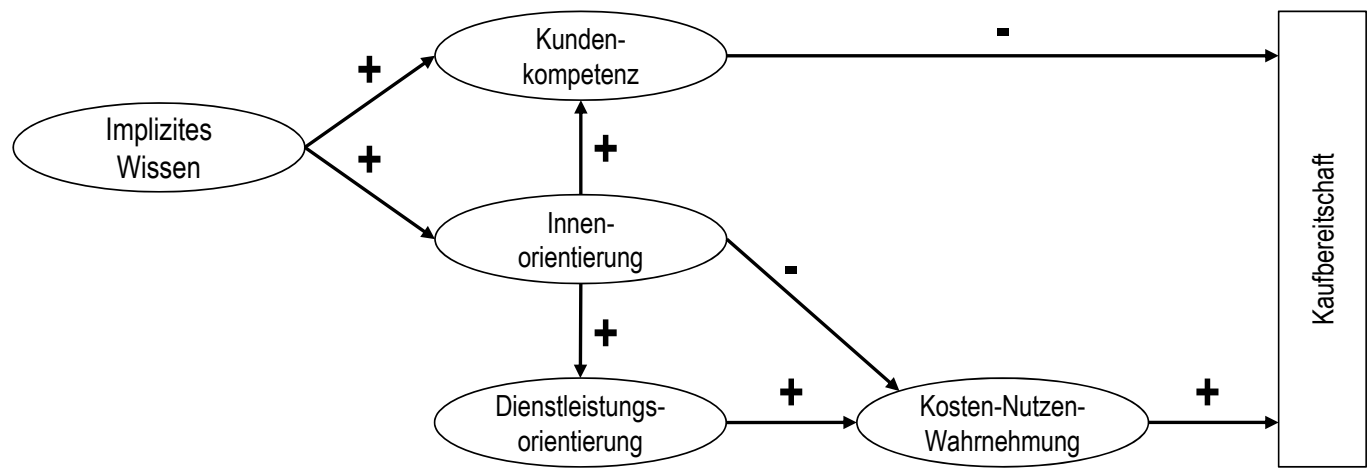

Abbildung 1: Modell für Kaufverhalten produktbegleitender Dienstleistungen

Erstens zeigt die Studie die grosse Bedeutung der unterschiedlichen Wissensarten, die für die Nutzung industrieller Güter und Dienstleistungen notwendig sind, für das erfolgreiche Angebot von produktbegleitenden Dienstleistungen. Das für die Leistungserbringung notwendige implizite Wissen fördert einerseits den Aufbau einer innenorientierten Unternehmenskultur, andererseits führt es dazu, dass der Kunde seine eigene Kompetenz in Relation zur Kompetenz des Herstellers als höher wahrnimmt. Implizites Wissen bezieht sich in diesem Zusammenhang sowohl auf die Kenntnis der betrieblichen Einbindung von beispielsweise Maschinen als auch, insbesondere bei Sondermaschinen, auf nicht dokumentierte Aspekte bezüglich ihrer Funktionsweise. Bei einem hohen Anteil impliziten Wissens ist dieses Wissen des Kunden oft wichtiger als das überlegene Produktwissen des Herstellers, wodurch die Kundenkompetenz in Relation höher erscheint. Damit wirkt sich ein hoher Anteil impliziten Wissens negativ auf die Neigung der Kunden aus, produktbegleitende Dienstleistungen zu kaufen. Da ein hoher Anteil an implizitem Wissen den Einweisungsaufwand des externen Personals erhöht, veranlasst dies Kunden zudem dazu, sich stärker nach innen zu orientieren. Die damit verbundene schlechtere Beurteilung des Kosten-Nutzen-Verhältnisses der angebotenen Dienstleistungen führt ebenfalls zu einer geringeren Kaufneigung der Kunden.

Zweitens zeigen die Ergebnisse, dass die Kosten-Nutzen-Wahrnehmung des Dienstleistungsangebots primär von der Unternehmenskultur des Kunden abhängt. Diesbezüglich 
wirkt die Innenorientierung der Kunden negativ, die Dienstleistungsorientierung dagegen positiv auf die Kosten-Nutzen-Wahrnehmung produktbegleitender Dienstleistungen.

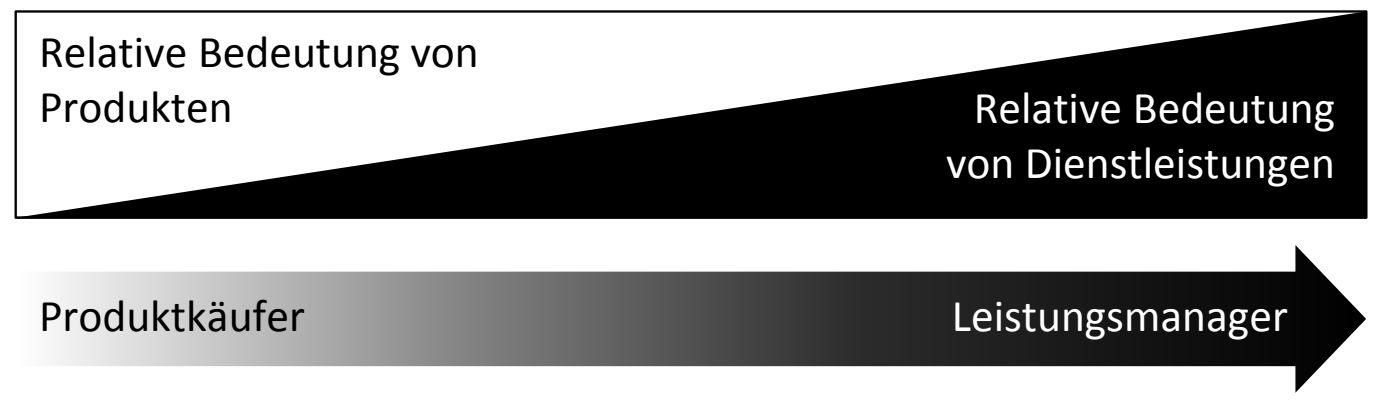

\section{Abbildung 2: Kundenseitiger Transitionsprozess}

Nach Oliva und Kallenberg (2003) durchlaufen Hersteller einen Transitionsprozess vom Produkthersteller zum Dienstleister. Aus der Analyse der Ergebnisse lässt sich schliessen, dass sich auch die Kunden entlang einer Transitionslinie vom reinen Produktkäufer zum Leistungsmanager entwickeln (vgl. Abbildung 2). Produktkäufer sind in diesem Zusammenhang primär am Erwerb des tangiblen industriellen Gutes interessiert, während für Leistungsmanager die Lösung einer Problemstellung durch Erwerb eines hybriden Angebots von Maschinen und Dienstleistungen im Vordergrund steht.

Drittens wird gezeigt, dass das Kaufverhalten von der Kundenkompetenz und der Wahrnehmung des Kosten-Nutzen-Verhältnisses des Dienstleistungsangebots abhängt. Besitzt der Kunde nicht das Wissen, um eine Leistung selbst zu erbringen, ist er zumeist auf den Bezug der entsprechenden Leistung vom Hersteller angewiesen. Sofern ein solches Abhängigkeitsverhältnis besteht, ist der Preis der Leistung für das Kaufverhalten der Kunden zweitrangig. Ist der Kunde hingegen nicht auf den Bezug der Leistung vom Hersteller angewiesen, so ist das wahrgenommene Kosten-Nutzen-Verhältnis des Angebots das entscheidende Kaufkriterium. Gängige Kundendienstleistungen, wie beispielsweise Reparaturen, lassen sich demnach unter geringem Preisdruck einfach vertreiben während sich die Absatzsituation für weiterführende Dienstleistungen meist schwerer darstellt. Hierin kann eine weitere Erklärung für das Auftreten des „Service Paradox“ gesehen werden.

\subsection{Implikationen für die Praxis}

Für die Unternehmenspraxis lassen sich aus den Erkenntnissen wichtige Rückschlüsse auf Erfolgsfaktoren für das Angebot produktbegleitender Dienstleistungen ziehen. Es kann gezeigt werden, dass der Verkaufserfolg bei produktbegleitenden Dienstleistungen stark vom fokussierten Kundensegment abhängt. Insbesondere stark innenorientierte Unternehmen ziehen den Kauf von Dienstleistungen eines Produktherstellers nur dann in Erwägung, wenn sie aufgrund fehlenden Produktwissens keine Möglichkeit haben, die Dienstleistung selbst zu erstellen. Anbieter sollten demnach die Entwicklung produktbegleitender Dienstleistungen primär auf Kundensegmente mit geringerer Innenorientierung ausrichten.

Die Ergebnisse zeigen zudem, dass implizites Wissen einen starken Einfluss auf das Kaufverhalten der Kundenunternehmen bezogen auf produktbegleitende Dienstleistungen hat. Ist für die Ausführung von Leistungen ein hoher Anteil impliziten Wissens erforder- 
lich, so bevorzugen Kunden eine Leistungserbringung durch eigenes Personal. In diesem Zusammenhang sind sowohl das implizite Wissen um die Einbindung der Maschine in kundenspezifische Prozesse als auch undokumentierte Spezifika der Maschine selbst von Relevanz für das Kaufverhalten. Demnach lassen sich Dienstleistungen für Standardmaschinen eher erfolgreich auf dem Markt etablieren als Dienstleistungen für hochspezifische Sondermaschinen, die eng in die Kundeninfrastruktur eingebunden sind.

Auch die Bedeutung der Dienstleistungsorientierung der Kunden für deren Kaufverhalten ist für Industriegüteranbieter von praktischer Relevanz. In der Studie kann gezeigt werden, dass Kundenunternehmen mit geringer Dienstleistungsorientierung nicht über Möglichkeiten verfügen, um Dienstleistungen des Herstellers mit selbsterbrachten Leistungen objektiv vergleichen zu können. Da die Kunden in der Folge meist die Kosten der Selbsterstellung von Leistungen unterschätzen, gestaltet sich der Verkauf von Dienstleistungen an Kunden mit geringer Dienstleistungsorientierung schwierig. Die Hersteller sollten diesbezüglich verstärkt den Ansatz des Value Bases Selling zum Einsatz bringen und gezielt die Vorteile der angebotenen Dienstleistung gegenüber der Selbsterstellung durch den Kunden darstellen (Terho et al. 2011).

Um erfolgreich Dienstleistungen anbieten zu können, ist der Hersteller darauf angewiesen, dass seine Kunden das Dienstleistungsangebot auf objektiver Basis mit selbst erbrachten Leistungen vergleichen. Die Erkenntnisse zeigen, dass sich Unternehmen bezüglich ihres Dienstleistungseinkaufs von reinen Produktkäufern zu Leistungsmanagern entwickeln (vgl. Abbildung 2). Um erfolgreich zu sein, sollten die Hersteller die Transitionsstufe ihrer Kunden kennen und ihr Angebot daran ausrichten. Sind die Dienstleistungsangebote zu komplex, kann dies zu einer geringen Marktakzeptanz führen. Ist das Dienstleistungsangebot hingegen nicht umfangreich genug, so vergeben Hersteller nicht nur Umsatzpotentiale, sondern riskieren auch einen Nachteil gegenüber Wettbewerbern.

\section{Limitationen und Ausblick}

Wie jede Forschungsarbeit weist auch die vorliegende Studie Limitationen auf, die auf zukünftige Forschungsgebiete hinweisen. So wurden in den Kundenunternehmen ausschliesslich zentrale Entscheidungsträger befragt. Entscheidungen über hochpreisige Dienstleistungsverträge werden jedoch zumeist von einem Buying Center getroffen, womit die vorliegende Studie die Sichtweise beratender Funktionen wie beispielsweise des Controllings ausser Acht lässt. Die Studie betrachtet zudem primär Kaufkriterien bezüglich eines Dienstleistungskaufs direkt vom Maschinenhersteller. Für die Teilnehmer waren Dienstleistungen von Drittanbietern zwar zumeist keine relevante Option, für ein vollständiges Bild und vor dem Hintergrund, dass die Erbringung eines Lösungsgeschäfts im Rahmen von Netzwerken oder Verbünden zunimmt, sollte zukünftige Forschung auch die Entscheidungssituation zwischen dem Hersteller und einem Drittanbieter mit einbeziehen. Eine weitere Einschränkung betrifft die Auswahl der Interviewpartner. Aufgrund der geringen theoretischen Fundierung des Forschungsgebietes wurde ein explorativ qualitativer Forschungsansatz gewählt. Eine quantitative Bestätigung der gefundenen Erkenntnisse stellt damit einen möglichen nächsten Schritt dar, um das hier vorgeschlagene Model auch empirisch validieren zu können. 


\section{Literaturhinweise}

Antioco, M./Moenaert, R.K./Lindgreen, A./Wetzels, M. (2008): Organizational antecedents to and consequences of service business orientations in manufacturing companies, in: Journal of the Academy of Marketing Science, Jg. 36, Nr. 3, S. 337-358.

Argyres, N. (1996): Evidence on the role of firm capabilities in vertical integration decisions, in: Strategic Management Journal, Jg. 17, Nr. 2, S. 129-150.

Bandler, J./Burke, D. (2012): How Hewlett-Packard lost its way, in: Fortune, Jg. 165, Nr. 7, S. 71-83.

Baveja, S./Gilbert, J./Ledingham, D. (2004): From products to services: why it's not so simple, in: Harvard Management Update, Jg. 9, Nr. 4, S. 3-6.

Corbin, J.M./Strauss, A.L. (2008): Basics of qualitative research: Techniques and procedures for developing grounded theory, 3. Aufl., Thousand Oaks.

Du, R.Y./Kamakura, W./Mela, C. (2007): Size and share of customer wallet, in: Journal of Marketing, Jg. 71, Nr. 2, S. 94-113.

Eggert, A./Hogreve, J./Ulaga, W./Muenkhoff, E. (2011): Industrial services, product innovations, and firm profitability: A multiple-group latent growth curve analysis, in: Industrial Marketing Management, Jg. 40, Nr. 5, S. 661-670.

Eggert, A./Hogreve, J./Ulaga, W./Muenkhoff, E. (2014): Revenue and profit implications of industrial service strategies, in: Journal of Service Research, Jg. 16, Nr. 4, S. 1-17.

Fang, E. (Er)/Palmatier, R.W./Steenkamp, J.-B.E. (2008): Effect of Service Transition Strategies on Firm Value, in: Journal of Marketing, Jg. 72, Nr. 5, S. 1-14.

Ganesh, J./Arnold, M./Reynolds, K. (2000): Understanding the customer base of service providers: an examination of the differences between switchers and stayers, in: The Journal of Marketing, Jg. 64, Nr. 3, S. 65-87.

Gebauer, H./Edvardsson, B./Gustafsson, A./Witell, L. (2010): Match or Mismatch: Strategy-Structure Configurations in the Service Business of Manufacturing Companies, in: Journal of Service Research, Jg. 13, Nr. 2, S. 198-215.

Gebauer, H./Fleisch, E./Friedli, T. (2005): Overcoming the Service Paradox in Manufacturing Companies, in: European Management Journal, Jg. 23, Nr. 1, S. 14-26.

Grant, R. (1996): Toward a knowledge-based theory of the firm, in: Strategic Management Journal, Jg. 17, Nr. 2, S. 109-122.

Homburg, C./Rudolph, B. (2001): Customer satisfaction in industrial markets: dimensional and multiple role issues, in: Journal of Business Research, Jg. 52, Nr. 1, S. 15-33.

Kogut, B./Zander, U. (1992): Knowledge of the Firm, Combinative Capabilities, and the Replication of Technology, in: Organization Science, Jg. 3, Nr. 3, S. 383-397.

Kunz, W.H./Hogreve, J. (2011): Toward a deeper understanding of service marketing: The past, the present, and the future, in: International Journal of Research in Marketing, Jg. 28, Nr. 3, S. 231-247.

Oliva, R./Kallenberg, R. (2003): Managing the transition from products to services, in: International Journal of Service Industry Management, Jg. 14, Nr. 2, S. 160-172.

Paulssen, M./Birk, M.M. (2007): Satisfaction and repurchase behavior in a business-to-business setting: Investigating the moderating effect of manufacturer, company and demographic characteristics, in: Industrial Marketing Management, Jg. 36, Nr. 7, S. 983-997. 
Raddats, C./Easingwood, C. (2010): Services growth options for B2B product-centric businesses, in: Industrial Marketing Management, Jg. 39, Nr. 8, S. 1334-1345.

Reinartz, W. J./Ulaga, W. (2008): How to sell services more profitably, in: Harvard Business Review, Jg. 86, Nr. 5, S. 90-96.

Terho, H./Haas, A./Eggert, A./Ulaga, W. (2011): “It”s almost like taking the sales out of selling'Towards a conceptualization of value-based selling in business markets, in: Industrial Marketing Management, Jg. 41, Nr. 1, S. 174-185.

Jens Hogreve, Prof. Dr., ist Professor für ABWL und Dienstleistungsmanagement an der Ingolstadt School of Management der Katholischen Universität Eichstätt-Ingolstadt.

Anschrift: Katholische Universität Eichstätt-Ingolstadt, Lehrstuhl für ABWL und Dienstleistungsmanagement, Auf der Schanz 49, D-85049 Ingolstadt, Tel.: +49 (0)841/937-21861, Fax: +49 (0)841/937-218600, E-Mail: jens.hogreve@ku.de

Richard Wonner, Dipl.-Wirt.-Ing., ist Mitarbeiter der AGCO Fendt GmbH und externer Doktorand am Lehrstuhl ABWL und für Dienstleistungsmanagement der Katholischen Universität Eichstätt-Ingolstadt.

Anschrift: Agnes-Wyssach-Str. 3a, D-87437 Kempten, Tel.: +49 (0)831/9606514, E-Mail: richard.wonner@ku.de 